\title{
Démographie, climat et alimentation mondiale
}

RAPPORT SUR LA SCIENCE ET LA TECHNOLOGIE № 32

Animateurs : Henri Leridon et Ghislain de Marsily

ACADÉMIE DES SCIENCES

(ए)

EDP

SCIENCES

17, avenue du Hoggar

Parc d'activités de Courtabœuf, BP 112

91944 Les Ulis Cedex A, France 
- Sciences et pays en développement. Afrique subsaharienne francophone RST n²1, 2006.

- La recherche spatiale française RST n²2, 2006.

- L'épidémiologie humaine. Conditions de son développement en France, et rôle des mathématiques

RST n²3, 2006.

- La maîtrise des maladies infectieuses. Un défi de santé publique, une ambition médico-scientifique

RST n²4, 2006.

- Les eaux continentales

RST n²5, 2006.

- La fusion nucléaire : de la recherche fondamentale à la production d'énergie?

RST n²6, 2006.

- Cycles biogéochimiques et écosystèmes continentaux RST n²7, 2007.

- Hormones, santé publique et environnement

RST n²8, 2008.

- Événements climatiques extrêmes. Réduire les vulnérabilités des systèmes écologiques et sociaux

RST n²9, 2010.

- Les sciences spatiales. Adapter la recherche française aux enjeux de l'Espace

RST n 30, 2010.

- La Métallurgie, science et ingénierie

RST n³1, 2010.

\section{Imprimé en France}

(C) 2011, EDP Sciences, 17, avenue du Hoggar, BP 112, Parc d'activités de Courtabœuf, 91944 Les Ulis Cedex A

Tous droits de traduction, d'adaptation et de reproduction par tous procédés réservés pour tous pays. Toute reproduction ou représentation intégrale ou partielle, par quelque procédé que ce soit, des pages publiées dans le présent ouvrage, faite sans l'autorisation de l'éditeur est illicite et constitue une contrefaçon. Seules sont autorisées, d'une part, les reproductions strictement réservées à l'usage privé du copiste et non destinées à une utilisation collective, et d'autre part, les courtes citations justifiées par le caractère scientifique ou d'information de l'œuvre dans laquelle elles sont incorporées (art. L. 122-4, L. 122-5 et L. 335-2 du Code de la propriété intellectuelle). Des photocopies payantes peuvent être réalisées avec l'accord de l'éditeur. S'adresser au : Centre français d'exploitation du droit de copie, 3, rue Hautefeuille, 75006 Paris. Tél. : 0143269535. 